\title{
Robotic Assisted MRI-US Fusion Guided Target Saturation Biopsy of the Prostate - Diagnostic Accuracy and Clinical Implications
}

Christian Wetterauer ( $\nabla$ christian.wetterauer@usb.ch )

University Hospital of Basel

Pawel Trotsenko

University Hospital of Basel

Marc Matthias

University Hospital of Basel

Christian Breit

University Hospital of Basel

Nicola Keller

University of Basel

Anja Meyer

University Hospital of Basel

Philipp Brantner

University Hospital of Basel

Tatjana Vlajnic

University Hospital of Basel

Lukas Bubendorf

University Hospital of Basel

David Winkel

University Hospital of Basel

Maciej Kwiatkowski

Kantonsspital Aarau

Helge Seifert

University Hospital of Basel

\section{Research Article}

Keywords: prostate biopsy, robotic-assisted, transperineal, target saturation

Posted Date: June 24th, 2021

DOl: https://doi.org/10.21203/rs.3.rs-633736/v1 
License: (c) (i) This work is licensed under a Creative Commons Attribution 4.0 International License. Read Full License 


\section{Abstract}

MRI-targeted prostate biopsy improves detection of clinically significant prostate cancer (PCa). However, up to $70 \%$ of PCa lesions display intralesional tumor heterogeneity. Current target sampling strategies do not yet adequately account for this finding. This prospective study included 118 patients who underwent transperineal robotic assisted biopsy of the prostate. We identified a total of 58 PCa-positive PI-RADS lesions. We compared diagnostic accuracy of a target-saturation biopsy strategy to accuracy of single, two, or three randomly selected targeted biopsies and analysed potential clinical implications.

Intralesional detection of clinically significant cancer (ISUP $\geq 2$ ) was $78.3 \%$ for target-saturation biopsy and $39.1 \%, 52.2 \%$, and $67.4 \%$ for one, two, and three targeted cores, respectively. Target-saturation biopsies led to a more accurate characterization of PCa in terms of Gleason score and reduced rates of missed cancer. Compared to one, two, and three targeted biopsies, target-saturation biopsies led to intensified staging procedures in $21.7 \%, 10.9$, and $8.7 \%$ of patients, and ultimately to a potential change in therapy in $39.1 \%, 26.1 \%$, and $10.9 \%$ of patients.

This work presents the concept of robotic-assisted target saturation biopsy. This technique has the potential to improve diagnostic accuracy and thus individual staging procedures and treatment decisions.

\section{Introduction}

Worldwide, prostate cancer (PCa) is the second most common cancer in men, and the second most common cause of cancer deaths ${ }^{1}$. Both incidence and mortality of PCa correlate with increasing age, with the average age at diagnosis being 66 years $^{2}$. Elevated prostate specific antigen (PSA) values and suspicious lesions in magnetic resonance imaging (MRI) can indicate the presence of PCa in these men ${ }^{1}$ but in order to definitely confirm the presence of PCa, a tissue sample must be taken. Multiple approaches and techniques for prostate biopsy have been described ${ }^{3}$. However, PCa displays a vast heterogeneity in terms of morphological and spatial heterogeneity, and contemporary biopsy regimens preferentially sample the peripheral zone ${ }^{4,5}$. Saturation biopsy concepts aim to detect and map any carcinoma, but harbour the risk of over-detecting clinically insignificant $\mathrm{PCa}^{5}$ and of complications ${ }^{6}$. MRItargeted approaches were shown to reduce over-detection and improve detection of clinically significant cancer ${ }^{7}$, and thus currently represent state of the art, even though systemic biopsy should not be omitted ${ }^{8,9}$. However, up to $70 \%$ of PCa lesions display intralesional tumor heterogeneity ${ }^{10}$, and targeted biopsy strategies do not yet adequately account for this finding, as the number of targeted biopsies varies significantly ${ }^{11-14}$ and no standard has been defined. Some authors have assessed the impact of the number of targeted biopsy per lesion ${ }^{11,12,15}$, and the combination of sampling the center and the periphery has been proposed ${ }^{10}$. However, there are no reports of targeted saturation biopsy strategies to specifically cover a lesion comprehensively. Noteworthy, under-sampling of suspicious lesion may pretend "false peace" and, in the worst case, ultimately lead to inadequate treatment decisions. 
Contemporary robotic-assisted biopsy techniques allow to perform prostate biopsies with upmost precision in an automated fashion and facilitate exact planning and execution of biopsy strategies in a 2dimensional and a 3 -dimensional fashion ${ }^{16}$. This technique provides the prerequisites to test the diagnostic yield of a new target saturation biopsy strategy in terms of providing representative samples of suspicious lesions for accurate identification and classification of PCa. This study aims to assess the potential of robotic-assisted target saturation biopsies in terms of intra-lesional diagnostic accuracy as well as its potential clinical implications.

\section{Materials And Methods}

\section{Patients}

For this prospective study, we analysed the results of 118 patients who had presented with elevated PSA values or suspicious lesions in MRI, and had undergone transperineal robotic-assisted biopsy of the prostate at the University Hospital Basel between January 2020 and May 2021. All patients provided written informed consent. The study was approved by the local ethics committee (ID 2020 - 01381), and was performed in accordance with the Declaration of Helsinki. Demographic, clinical, and histological data were recorded and analysed. All PCa-positive PI-RADS lesions sampled by targeted saturation biopsies were identified. Lesions $>3 \mathrm{ml}$ were excluded from the analysis. A total of 58 PCa-positive PIRADS lesions in 46 patients were included in this study.

3-D Modeling, equipment, biopsy technique and histological analysis

At our institution, a skilled team of radiologists (DJW, PB) classified all suspicious lesions according to PI-RADS v2.1, manually contoured the prostate including lesions, and generated a 3D model (Urofusion, Biobot()). Target lesion volume was calculated automatically by the software. All robotic-assisted targeted biopsies of the prostate were performed with an iSR'obot ${ }^{\mathrm{TM}}$ MonaLisa device (Biobot $\odot$ ) by one experienced surgeon (CW). The first 60 (50.8\%) patients in this cohort received antibiotic prophylaxis. We later principally abstained from antibiotic prophylaxis if not indicated otherwise. All biopsies were performed under general anesthesia. The Mona Lisa system was connected to an ultrasound scanner (Specto, BK Medical ${ }^{\circledR}$ ) with a transrectal probe (BK Medical $\left.{ }^{\circledR}\right)$. All patients underwent either random biopsies in combination with targeted biopsies or targeted biopsies only in within the framework of a MRI based screening study. Either way, the number of random and targeted biopsies were planned softwaresupported according to prostate and lesion size (Urobiopsy, Biobot@). All targeted biopsies were optimized manually according to individual shape of the lesion, pursuing a target saturation biopsy strategy in order to gain representative sampling from center and peripheries. Biopsy density was adjusted according to a reasonable lesionvolume adapted approach. Detailed information on biopsy density is displayed in Table 1. A reusable biopsy gun with trocar-shaped biopsy needles (Uromed@) or single-use biopsy needles (Bard@) were used to gain histological samples through two incision points. Every biopsy position was controlled with realtime-ultrasound and every single biopsy core was placed in a separate box and collected in formalin for further processing. Histological evaluation was performed by 
specialized urological pathologist (TV, LB), including positive percentage of tumor, length of tumor tissue, Gleason patterns, ISUP-Grade groups, and perineural invasion for each biopsy-core. Incisions were covered with sterile plasters. The patients received no transurethral catheter. The detailed procedure has been described previously ${ }^{16,17}$.

Table 1

Baseline characteristics

\begin{tabular}{|lll|}
\hline Parameter & Total $(\mathrm{n})$ & Mean \pm SD (range) \\
\hline Patients & 46 & - \\
\hline Age $($ years $)$ & - & $67 \pm 6.8(50.9-84)$ \\
\hline Prostate volume $\left(\mathrm{cm}^{3}\right)$ & - & $42.7 \pm 17.4(14-87)$ \\
\hline Total PSA $(\mathrm{ng} / \mathrm{ml})$ & - & $11.1 \pm 18.3(1.1-109)$ \\
\hline PSA density $\left(\mathrm{ng} / \mathrm{ml}^{2}\right)$ & - & $0.3 \pm 0.5(0.05-3)$ \\
\hline Positive lesions & 58 & - \\
\hline PI-RADS III & 9 & - \\
\hline PI-RADS IV & 37 & - \\
\hline PI-RADS V & 12 & - \\
\hline Lesion volume $(\mathrm{ml})$ & - & $0.7 \pm 0.6(0.1-2.7)$ \\
\hline Number of biopsies per lesion & - & $6.2 \pm 1.8(3-11)$ \\
\hline Biopsy density $(1 / \mathrm{ml})$ for all lesions & 58 & $14.9 \pm 10.4(2.6-50)$ \\
\hline Biopsy density $(1 / \mathrm{ml})$ for lesions $<1 \mathrm{ml}$ & 42 & $18.4 \pm 10.1(7.3-50)$ \\
\hline Biopsy density $(1 / \mathrm{ml})$ for lesions $\geq 1 \mathrm{ml}$ & 16 & $5.5 \pm 1.8(2.6-9.2)$ \\
\hline $\begin{array}{l}\text { SD, standard deviation; } P S A \text {, prostate-specific antigen; } \\
\text { system }\end{array}$ & & \\
\hline
\end{tabular}

Analysis and statistical methods

We analysed the histopathologic results of all targeted biopsies $\left(B_{x}\right)$ taken from lesions in which cancer was detected, and calculated the diagnostic yield on a per-lesion level and on a per-patient level. In case of multiple positive PI-RADS lesions per patient, a main lesion was defined, primarily based on the highest ISUP grade detected and secondarily based on the number of positive biopsies. We compared the diagnostic accuracy of a single targeted biopsy, of two targeted biopsies, and of three targeted biopsies to the results of target-saturation biopsies $\left(\mathrm{B}_{\text {sat }}\right)$ in MRI-visible lesions. For the three strategies mentioned first, randomly selected targeted biopsy cores were analysed. Furthermore, we defined a worst-case scenario in which we considered either tumor-free biopsies or the lowest ISUP grade group core detected 
within the lesion in case of all positive biopsies, and compared this to results of target-saturation biopsies. In order to assess the clinical implications of the respective biopsy strategies, we analysed the number of cancers missed, the number of cancers with Gleason upgrading, potential treatment alteration (active surveillance instead of PSA monitoring or switch to curative treatment due to ISUP grade $>1$ ), as well as the number of insufficient staging (ISUP grade $>2$ ) both on a per-lesion and a per-patient level. Statistical analyses were performed with SPSS Statistics 24.0 (IBMC), and the database was created using Excel (MicrosoftC). All tests were performed at a two-sided significance level of $a=0.05$.

\section{Results}

Transperineal robotic-assisted biopsy of the prostate (TP-RA-PB $_{\mathrm{x}}$ ) was successfully performed in 103 patients with suspicious lesions. We identified a total of 58 PCa-positive PI-RADS lesions $(<3 \mathrm{ml})$ in 46 patients sampled by targeted saturation biopsies. A flowchart of the study course (enrolment and inclusion) is presented in Fig. 1 according to the TREND statement. Mean (range) age and PSA were $67(50.9-84)$ years and $11.1(1.1-109) \mathrm{ng} / \mathrm{ml}$, respectively. Mean (range) target lesion volume was 0.7 $(0.1-2.7) \mathrm{ml}$. Detailed patient baseline characteristics are summarized in Table 1.

The rate of patients with clinically significant cancer (ISUP grade $\geq 2$ ) based on one, two, and three cores, or on target-saturation biopsy was $39.1 \%$ (18/46), 52.2\% (24/46), 67.4\% and 78.3\% (36/46), respectively. The rates of clinically significant disease on lesion level based on the respective biopsy strategy were $36.2 \%(21 / 58), 51.7 \%$ (30/58), 65.5\% (38/58), and 77.6\% (45/58), respectively. On both lesion and patient level, target-saturation biopsies led to higher ISUP grades and significantly reduced rates of missed cancer as compared to one and two targeted biopsy cores (Table 2). Compared to one, two, and three targeted biopsies, target-saturation biopsies led to change in therapy (indication ISUP grade $>1$ ) in 18 (39.1\%), $12(26.1 \%)$, and $5(10.9 \%)$ of the patients, respectively. In comparison to target-saturation biopsies, staging procedures in the groups of patients with one, two, and three targeted biopsies were insufficient in $10(21.7 \%), 5(10.9 \%)$, and $4(8.7 \%)$ of the patients. Detailed results comparing the diagnostic accuracy and clinical implications of all biopsy strategies as well as for the worst case scenario are displayed on both lesion and patient level in Table 2 and supplementary Table 1. 
Table 2

Comparison of biopsy strategies on lesion and patient level

\begin{tabular}{|c|c|c|c|c|c|c|}
\hline \multirow{2}{*}{$\begin{array}{l}\text { Parameter } \\
\text { RS - Lesions } n=58\end{array}$} & \multicolumn{3}{|c|}{$1 B_{x}$ vs. $B_{\text {sat }}$} & \multicolumn{2}{|c|}{$3 B_{x}$ vs. $B_{\text {sat }}$} & \multirow[b]{2}{*}{$p$} \\
\hline & $\mathrm{n}(\%)$ & $\mathrm{p}$ & $\mathrm{n}(\%)$ & $\mathrm{p}$ & $\mathrm{n}(\%)$ & \\
\hline Cancer missed & $21(36.2)$ & 0.003 & $12(20.7)$ & 0.018 & $6(10.3)$ & 0.07 \\
\hline Gleason upgrade & $34(58.6)$ & - & $22(37.9)$ & - & $12(20.7)$ & - \\
\hline Change in definitive treatment ${ }^{1}$ & $24(41.4)$ & $<0.001$ & $15(25.9)$ & 0.004 & $7(12.1)$ & 0.15 \\
\hline Insufficient staging ${ }^{2}$ & $11(19.0)$ & 0.028 & $5(8.6)$ & 0.3 & $3(5.2)$ & 0.56 \\
\hline RS - Patients $n=46$ & $\mathrm{n}(\%)$ & $\mathrm{p}$ & $\mathrm{n}(\%)$ & $\mathrm{p}$ & $\mathrm{n}(\%)$ & $\mathrm{p}$ \\
\hline Cancer missed & $17(37.0)$ & 0.006 & $10(21.7)$ & 0.03 & $5(10.9)$ & 0.09 \\
\hline Gleason upgrade & $28(60.9)$ & - & $19(41.3)$ & - & $10(21.7)$ & - \\
\hline Change in definitive treatment ${ }^{1}$ & $18(39.1)$ & $<0.001$ & $12(26.1)$ & 0.01 & $5(10.9)$ & 0.24 \\
\hline Insufficient staging ${ }^{2}$ & $10(21.7)$ & 0.026 & $5(10.9)$ & 0.28 & $3(6.5)$ & 0.52 \\
\hline WCS - Lesions $\mathrm{n}=58$ & $\mathrm{n}(\%)$ & $\mathrm{p}$ & $\mathrm{n}(\%)$ & $\mathrm{p}$ & $\mathrm{n}(\%)$ & $\mathrm{p}$ \\
\hline Cancer missed & $35(60.3)$ & $<0.001$ & $26(44.8)$ & 0.002 & $17(29.3)$ & 0.007 \\
\hline Gleason upgrade & $50(86.2)$ & - & $45(77.6)$ & - & $35(60.3)$ & - \\
\hline Change in definitive treatment ${ }^{1}$ & $33(56.9)$ & $<0.001$ & $28(48.3)$ & $<0.001$ & $20(34.5)$ & $<0.001$ \\
\hline Insufficient staging ${ }^{2}$ & $18(31.0)$ & $<0.001$ & $16(27.6)$ & 0.001 & $12(20.7)$ & 0.02 \\
\hline WCS - Patients $n=46$ & $\mathrm{n}(\%)$ & $\mathrm{p}$ & $\mathrm{n}(\%)$ & $\mathrm{p}$ & $\mathrm{n}(\%)$ & $\mathrm{p}$ \\
\hline Cancer missed & $28(60.9)$ & $<0.001$ & $21(45.7)$ & 0.002 & $15(32.6)$ & 0.008 \\
\hline Gleason upgrade & $41(89.1)$ & - & $36(78.3)$ & - & $28(60.9)$ & - \\
\hline Change in definitive treatment ${ }^{1}$ & $25(54.3)$ & $<0.001$ & $21(45.7)$ & $<0.001$ & $17(37.0)$ & $<0.001$ \\
\hline Insufficient staging ${ }^{2}$ & $15(32.6)$ & $<0.001$ & $14(30.4)$ & 0.002 & $11(23.9)$ & 0.01 \\
\hline \multicolumn{7}{|c|}{$B_{x}$, target biopsy; $B_{\text {sat }}$ saturation biopsy; $R S$, random selection; $W C S$, worst case scenario } \\
\hline${ }^{1}$ Definition: Definitive $t$ & coraing & easor & re $(>6)$ & ated. & & \\
\hline
\end{tabular}

\section{Discussion}


This work is the first to assess the diagnostic accuracy of robotic-assisted transperineal target saturation biopsies of the prostate. The target saturation strategy aims to provide representative samples of suspicious lesions for upmost accurate identification and classification of PCa (Fig. 2). Cancerous lesions are known to display intralesional tumor heterogeneity in up to $70 \%$ of the cases ${ }^{10}$, and target saturation biopsies may represent the best strategy to reflect intralesional heterogeneity. Our data indicate that this approach is superior in terms of detection of clinically significant cancer as compared to up to three targeted biopsies ( $78.3 \%$ vs $67.4 \%)$. Some authors claim that two to three biopsy cores may be sufficient in PI-RADS 4 and 5 lesions ${ }^{15}$. However, our data indicate that the application of target saturation biopsies - with a median number of 6 biopsies per lesion in this study - can reduce the number of missed cancer in a statistically significant manner, and can provide a more accurate characterization in terms of assigned Gleason score for PCa detected within the lesion. Moreover, these results would both alter treatment decisions in a relevant number of patients and improve their clinical staging by more adequate risk stratification (Table 2). Furthermore, we present data for a "worst case" scenario that can be deemed valuable to assist the decision making process and patient counseling.

Our study has its limitations due to the single center data, the limited number of patients, and the single surgeon experience. The "random selection" scenario was applied to limit potential bias. However, we presume that this scenario may not reflect a completely adequate diagnostic accuracy of the respective biopsy strategy, which needs to be evaluated in a randomized trial. Further research is needed to decipher the impact of targeted biopsies taken from the center of a lesion and the peripheries, as well as the diagnostic performance in relation to PI-RADS score. Correlation of biopsy findings with prostatectomy specimen is required in a prospective setting in order to provide more evidence for the diagnostic accuracy of targeted saturation biopsies.

Especially in negative lesions, the additional morbidity caused by higher number of biopsies ${ }^{6}$ needs to be considered and weighed against the potentially improved diagnostic accuracy in positive lesions. Noteworthy, this study does not aim to assess or compare concepts of random biopsies or saturation biopsies for whole gland sampling. Rather, this work presents the concept of robotic-assisted target saturation biopsy, which was developed to provide representative samples from MRI-detected lesions with respect to tumor heterogeneity ${ }^{10}$. The robotic assisted approach enables high precision biopsy and the target saturation sampling approach provides complete diagnostic coverage of lesions.

Overall, this work indicates that the "target adapted saturation strategy" may be superior to predefined biopsy numbers (at least in the range between one and three targeted biopsies), and seems to more adequately reflect intralesional tumor heterogeneity. Our findings highlight the potential of roboticassisted target saturation biopsy in terms of correct classification, staging, and treatment decision, especially if a MRI-targeted strategy is pursued ${ }^{7}$. Well-designed future studies are needed to confirm these preliminary findings.

\section{Conclusions}


This work presents the concept of robotic-assisted MRI-US fusion guided target saturation biopsy of the prostate. The robotic assisted approach enables high precision biopsy and the target saturation sampling approach provides representative diagnostic coverage of lesions. This technique has the potential to improve diagnostic accuracy and thus individual staging procedures and treatment decisions.

\section{Declarations}

Acknowledgements: The authors want to thank Dr. Selina Ackermann from the University Hospital Basel for editorial assistance and NK for creating the graphics.

Authors' contributions: All authors have conjointly designed the study, and CW and PT interpreted the data and drafted the manuscript. AM supported data collection and patient care. All authors designed and critically revised the manuscript for important intellectual content. PT and CW were involved in the statistical analysis. All authors read, gave comments, and approved the final version of the manuscript.

Funding: None.

Conflicts of interest: CW received research grants from Siemens Healthineers and Uromed. The rest of the authors declare that they have no conflict of interest.

Ethics approval: Approval by the local Ethics Committee was granted (Ethikkommission Nordwest- und Zentralschweiz; ID 2020-01381).

Consent to participate: All patients confirmed their participation in our study with a signed informed consent.

Availability of data and materials: All data are available from the corresponding author upon reasonable request.

\section{References}

1. Mottet, N. et al. EAU-ESTRO-SIOG Guidelines on Prostate Cancer. Part 1: Screening, Diagnosis, and Local Treatment with Curative Intent. Eur. Urol. 71, 618-629 (2017).

2. Rawla, P. Epidemiology of Prostate Cancer. World J Oncol 10, 63-89 (2019).

3. Wetterauer, C. et al. Feasibility of freehand MRI/US cognitive fusion transperineal biopsy of the prostate in local anaesthesia as in-office procedure-experience with 400 patients. Prostate Cancer Prostatic Dis 23, 429-434 (2020).

4. Tolkach, Y. \& Kristiansen, G. The Heterogeneity of Prostate Cancer: A Practical Approach. Pathobiology 85, 108-116 (2018).

5. Scattoni, V. et al. Extended and saturation prostatic biopsy in the diagnosis and characterisation of prostate cancer: a critical analysis of the literature. Eur Uro/ 52, 1309-1322 (2007).

6. Loeb, S. et al. Systematic review of complications of prostate biopsy. Eur Urol 64, 876-892 (2013). 
7. Kasivisvanathan, V. et al. MRI-Targeted or Standard Biopsy for Prostate-Cancer Diagnosis. N Engl J Med 378, 1767-1777 (2018).

8. Ahdoot, M. et al. MRI-Targeted, Systematic, and Combined Biopsy for Prostate Cancer Diagnosis. N Engl J Med 382, 917-928 (2020).

9. Miah, S. et al. A prospective analysis of robotic targeted MRI-US fusion prostate biopsy using the centroid targeting approach. J Robot Surg 14, 69-74 (2020).

10. Fujihara* Atsuko et al. Mp51-02 intra-lesional prostate cancer heterogeneity in step-sectioned radical prostatectomy specimens: implications for target biopsy strategy. Journal of Urology 203, e763-e764 (2020).

11. Leyh-Bannurah, S.-R. et al. Minimum Magnetic Resonance Imaging-Ultrasound Fusion Targeted Biopsy Cores Needed for Prostate Cancer Detection: Multivariable Retrospective, Lesion Based Analyses of Patients Treated with Radical Prostatectomy. J Urol 203, 299-303 (2020).

12. Hansen, N. L. et al. Optimising the number of cores for magnetic resonance imaging-guided targeted and systematic transperineal prostate biopsy. BJU Int 125, 260-269 (2020).

13. Lu, A. J. et al. Role of Core Number and Location in Targeted Magnetic Resonance ImagingUltrasound Fusion Prostate Biopsy. Eur Urol 76, 14-17 (2019).

14. Dimitroulis, P. et al. Multiparametric Magnetic Resonance Imaging/Ultrasound Fusion Prostate Biopsy-Are 2 Biopsy Cores per Magnetic Resonance Imaging Lesion Required? J Urol 200, $1030-$ 1034 (2018).

15. Sonmez, G., Demirtas, T., Tombul, S. T., Ozturk, F. \& Demirtas, A. What is the ideal number of biopsy cores per lesion in targeted prostate biopsy? Prostate Int 8, 112-115 (2020).

16. Lim, S. et al. Robotic Transrectal Ultrasound Guided Prostate Biopsy. IEEE Trans Biomed Eng 66, 2527-2537 (2019).

17. Kroenig, M. et al. Diagnostic Accuracy of Robot-Guided, Software Based Transperineal MRI/TRUS Fusion Biopsy of the Prostate in a High Risk Population of Previously Biopsy Negative Men. Biomed Res Int 2016, 2384894 (2016).

\section{Figures}




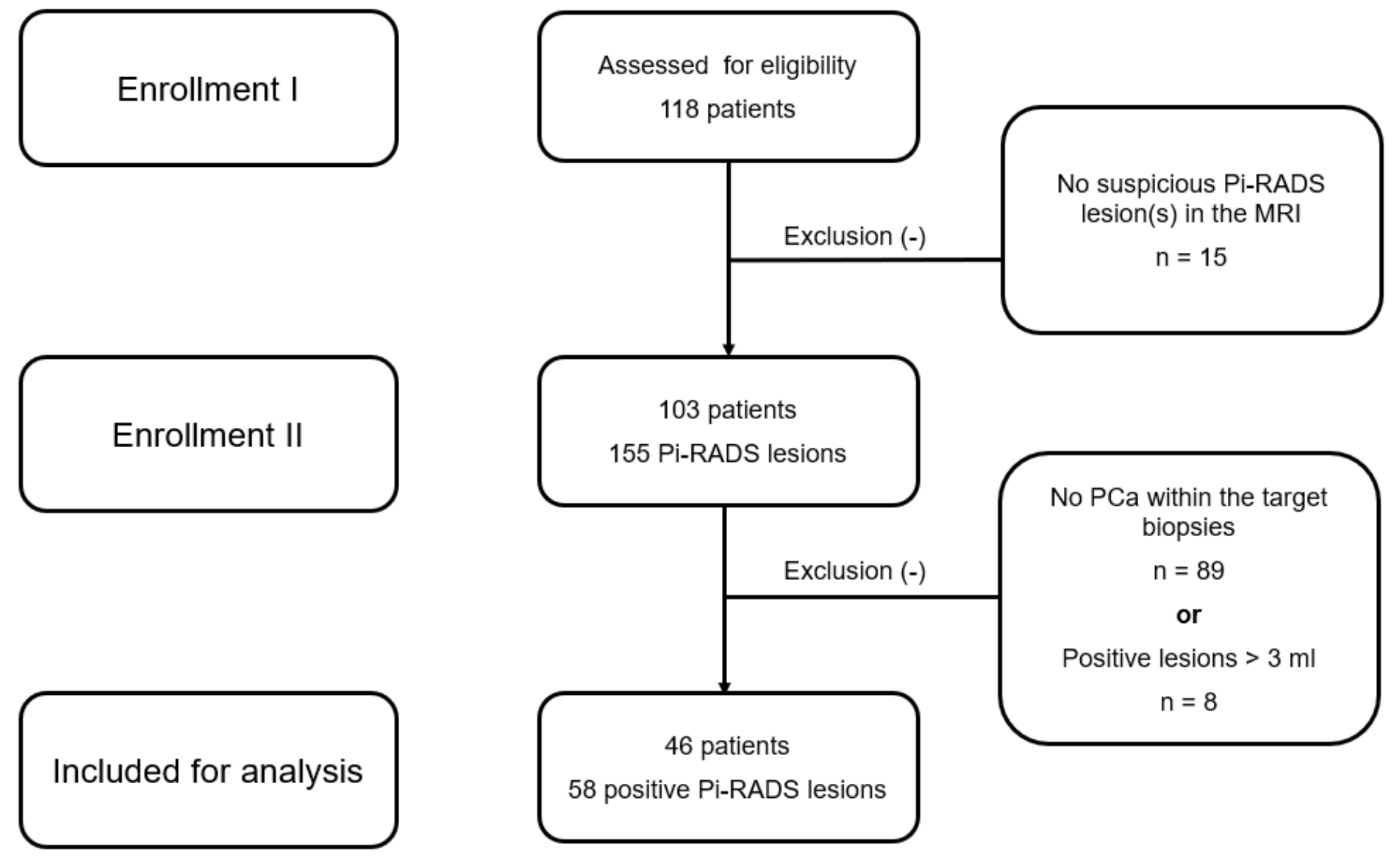

Figure 1

Flowchart of enrollment and inclusion according to TREND statement 


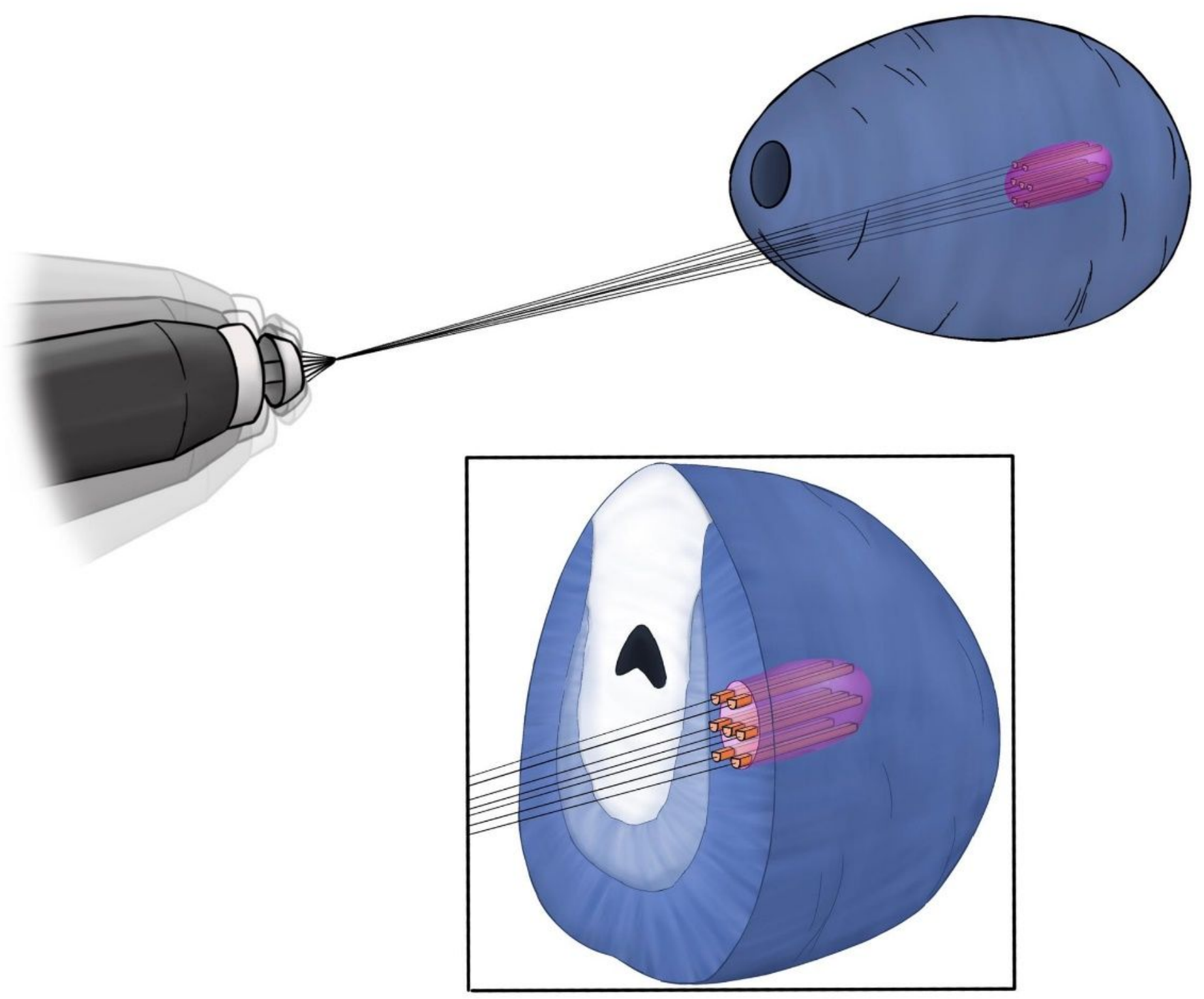

Figure 2

Concept of robotic assisted target saturation biopsy

\section{Supplementary Files}

This is a list of supplementary files associated with this preprint. Click to download.

- SupplementaryTable1Distributionofbiopsies58.docx 